\title{
Research on the Momentum Effect and Contrarian long-short portfolio base on CSI 800 Index
}

\author{
Zeng Jian ${ }^{1}$, a*
}

${ }^{1}$ School of Economics and Commerce, South China University of Technology, Guangzhou, Guangdong, China

azengjj111@163.com

Keywords: Quantitative investment; momentum; reversal; long-short strategy.

\begin{abstract}
In this paper, we use the data of CSI 800 constituent stocks from January 15, 2007 to June 29, 2012 to test the effectiveness of the method of dividend yield. At the same time, compare with the traditional method of dividing stock by equal amount, Different models are used to test the momentum and reversal effects of Chinese stock market. The model is modified by using 150/50 strategy, and the result is good.
\end{abstract}

\section{基于中证800指数对中国股市动量与反转多空组合的研究}

\author{
曾健 ${ }^{1, a^{*}}$ \\ 1华南理工大学经济与贸易学院, 广州, 广东, 中国 \\ azengjj111@163.com
}

关键词：量化投资；动量；反转；多空策略

中文摘要. 本文运用中证800成分股2007年1月15号到2012年6月29号的数据对等量划分收益 率方法的效果进行检验，同时与传统的按等量划分股票的方法做比较，并以不同的研究对象 来检测中国股市存在的动量与反转效应, 以此构造投资模型, 通过对模型进行修改, 运用多 空150/50策略, 取得了较好的收益效果。

\section{1. 引言}

已有的研究表明中国股市上存在着动量与反转的投资机会,国外最先发现股票市场中的 动量与反转效应分别是Jegadeesh和Titman (1993, 2001) 和De Bondt和Thaler (1985)。De Bondt 和Thaler(1985) 研究发现,在3年的组合形成期后，表现最差的10\%的股票所构成的投资组合 (输家)在3年持有期内的报酬超过了过去表现最好的 $10 \%$ 的股票所构成的投资组合(赢家), 而且 输家的报酬超过市场收益的 $19.6 \%$, 而赢家却比市场收益还低 $5.0 \%$ 。这一研究证实, 股票收益 反转现象在长窗口期内存在, 采取反转策略可以在较长期内获得可观的超常收益。Lehmann

(1990) 研究也表明, 在短期 (1个月之内) 股票收益也存在反转效应。而在国内, 王璞用2000 年到2009年沪深2000多只股票以月度为频率检验其动量与反转效应, 得出在持有期 6 个月内都 存在反转效应，且随着持有期的增长，投资组合收益率越来越低，反转现象越显著。最近几 年, 更是投研究者研究周期频率更短的反转效应, 严太华、梁岗研究分析得出在周数据中存 在反转效应, 这个发现与 $\mathrm{Li}, \mathrm{Pan}$ 等人所发现的大相径庭。实际上, 动量与反转效应检验也只 在特定的形成期和持有期中才存在，而且中国股市相对于其他发达国家而言并未成熟，投资 
者对于热点的切换及投资者的偏好会经常发生转变,短期投资远多于长期投资，这使得动量效 应跟反转效可能在一段时间内反复出现, 具有较多的投资机会。在我们研究动量与反转的现 象之上,同时考虑涉及多空，因为海外多空策略表现非常好，明显好于标普500，比对冲基金 还要好, 所以我们相信多空策略可能存在较为稳定的收益。SandyLee详细介绍了多空策略的 使用, 很有借鉴意义。而我们为什么要选择中证 800 作为研究对象, 是因为中证 800 在整合了 中证500和沪深300之后, 代表了沪深证券市场内大中小市值公司的整体状况, 能够较为全面 的代表宏观经济走势, 同时股票数目的相于沪深2000多只股票而言较少, 研究起来较为简单, 同时由于其代表性, 可能存在更多的交易机会。所以本文针对中证 800 进行研究, 而且数据更 新到2012年6月29日，同时对多个模型进行比较、修正，从而构造出更为有效的模型。

\section{2. 研究方法及数据选取}

本文选取从 2007 年 1 月 15 号到2012年6月 29 号的中证指数日收盘价数据, 所选择的样本期 的投资组合是根据以选定的样本期 (一周或一个月) 为标准的, 对样本期长度内各只股票的累 积收益率从小到大进行排序, 然后再持有一定的检验期长度 (一周或一个月), 记录其收益率, 第二次投资持有的股票是以上一期最后一天为终点, 往前推一个样本期长度对股票的累积收 益率进行排序为基准的, 然后再持有一定的检验期, 记录其收益率, 如此循环, 直至到 2012 年6月 29 号。当然, 这中间怎样划分中证800各成分股到各个分位, 就产生了我们检验的两种 模型:

a）等量划分股票为5分位; 这样的结果就是每个分位基本都有 160 只股票。

b）等量划分收益率为5分位; 将样本期的各股票的收益率最大跟最小的区间平均划 分为 5 个分位, 在此区间股票都将进入各自的分位, 每个分位的股票数都是随机 而定的。

在此基础上, 我们观察两个模型中收益率的情况, 并做相应的调整, 直至找到比较稳定, 且有较好收益的投资组合。

\section{3. 检验等量划分收益率方法的效果}

Li, Pan等人在《Weekly momentum by return interval ranking》一文中，运用1995-2005年沪 深所有股票的数据对Jegadeesh和 Titman (1993)中J-K策略的收益情况进行检验, 得出按等量 划分收益率为 5 分位的收益情况比传统的等量划分股票为5个分位的收益情况好。而我们基于 中证 800 , 分别对这两种模型进行检验, 选取 5,20天作为样本期的选择长度, 同样的选取 5,20 天作为检验期的持有长度。选择 5 天、 20 天作为时间长度, 是因为他们分别代表了一周跟一个 月的长度, 所以分别对不同时间长度进行匹配, 以初始资金为 1 按复利形式得出最后收益。两 种模型, 每种模型共有4组配对, 在总共这8组中, 其最终收益如下:

表1 等量划分股票为5分位时每个分位的最终收益率

\begin{tabular}{|l|c|c|c|c|c|}
\hline 样本期-持有期 & 第一分位 & 第二分位 & 第三分位 & 第四分位 & 第五分位 \\
\hline $5-5$ & 5.3949 & 3.7767 & 2.6427 & 1.2318 & 0.3435 \\
\hline $5-20$ & 1.6148 & 2.2735 & 2.7609 & 2.0352 & 0.9713 \\
\hline $20-5$ & 3.0729 & 2.3918 & 2.1502 & 1.4879 & 0.7212 \\
\hline $20-20$ & 2.9591 & 2.1855 & 2.0799 & 1.5040 & 0.9555 \\
\hline
\end{tabular}

表2 等量划分收益率为 5 分位时每个分位的最终收益率

\begin{tabular}{|l|r|r|r|r|c|}
\hline 样本期-持有期 & 第一分位 & 第二分位 & 第三分位 & 第四分位 & 第五分位 \\
\hline $5-5$ & 1.4829 & 2.4217 & 0.5636 & 0.3321 & 1.4485 \\
\hline $5-20$ & 0.8733 & 1.9955 & 1.0066 & 0.5304 & 0.4106 \\
\hline $20-5$ & 1.6277 & 1.2372 & 0.6462 & 0.2145 & 0.1902 \\
\hline $20-20$ & 3.4221 & 1.8604 & 0.8814 & 0.7168 & 1.9802 \\
\hline
\end{tabular}


表3 等量划分股票的最终收益率

\begin{tabular}{|l|c|c|c|c|c|}
\hline 样本期-持有期 & 第一分位 & 第二分位 & 第三分位 & 第西分位 & 位 \\
\hline $5-5$ & 7.799906 & 5.188677 & 3.737796 & 2.316893 & 1.881305 \\
\hline $5-20$ & 2.716359 & 3.129358 & 3.488131 & 2.673478 & 2.268887 \\
\hline $20-5$ & 4.171444 & 3.634327 & 3.248995 & 2.505812 & 2.165845 \\
\hline $20-20$ & 3.801182 & 2.930962 & 2.745661 & 2.362726 & 2.259856 \\
\hline
\end{tabular}

表4 等量划分收益率的最终收益率

\begin{tabular}{|l|c|c|c|c|c|}
\hline 样本期-持有期 & 第一分位 & 第二分位 & 第三分位 & 四分位 & 第五分位 \\
\hline $5-5$ & 3.792326 & 3.611517 & 1.824748 & 1.82444 & 3.702563 \\
\hline $5-20$ & 2.120451 & 2.737112 & 2.251513 & 2.209929 & 2.879851 \\
\hline $20-5$ & 2.612373 & 2.317962 & 1.838059 & 1.994396 & 1.646978 \\
\hline $20-20$ & 3.66819 & 2.446415 & 2.06358 & 1.828336 & 6.511795 \\
\hline
\end{tabular}

从第一个模型我们可以发现, 按等量划分股票的5-5策略第一分位取得了较好的收益, 这 个分位不仅存在期间收益最大值，也存在最后收益的最大值，但是其第五分位的收益为负数。 对于按等量划分收益率为五分位的20-20策略，其第一分位存在最后收益的最大值，但是期间 的最大值却是第五分位。我们之前定义划分进第一分位的股票是在样本期长度内累计收益率 最低的股票，他们在按等量划分股票5-5策略中在检验期收获不错的收益，在等量划分收益率 20-20策略中也存在的收益, 当相对较弱, 说明中国股市在短期存在较强的反转效应; 第五分 位定义为样本期收益率最好的分位组合，在按等量划分股票的5-5策略时收益为负，但是相对 亏损不大，说明依旧存在反转效应，但等量划分收益率20-20策略下检验期却能延续好的收益 效应, 说明中长期股市也存在动量效应。这与之前研究的中国投资者对于热点短期地切换及 投资者的偏好会经常发生转变相吻合, 说明在中国股市上存在短期的反转投资机会, 长期中 存在动量的投资机会。

同时, 我们可以看到 $\mathrm{Li}, \mathrm{Pan}$ 等人所构造的以等量划分收益率为5分位的模型在不同的选择 对象中并不一定优于将股票等量划分为 5 分位的模型。在传统的方法中, 我们将股票等量划分 为 5 个分位, 不仅有着期间收益的最大值, 还存在最终的最大值, 但 $\mathrm{Li}, \mathrm{Pan}$ 等人所提出的按等 量划分收益率为 5 个分位的模型, 在中证 800 中并没能表现出很好的投资收益, 以短期（即一 周）作为样本期长度选择投资组合，其第一分位的收益不如第二分位，与其在用95年至05年 用沪深A股的数据得出的结果截然相反, 当然我们并不能因此评价该模型不好, 毕竟选择的 研究对象不同，Li，Pan等人研究的是1995-2009年沪深A股的股票，它比中证800有着更多的投 资对象, 比如有更多中小板股票, 中小板由于其市值小而易被操控, 所以短期操作可能更为 频繁, 这也许才使得 $\mathrm{Li}, \mathrm{Pan}$ 等人所研究的模型得到不错的收益。而本文只是在次贷危机前后 2007-2012年中证800的数据, 即成分股都是大中市值的股票。虽然得出模型的优劣收益不同, 但是我们所要关注的是如何构造更好的稳定收益以应对动荡不止的宏观经济。显然, 上面两 种模型并不能符合我们的要求。我们是可以看到两种模型都能在一定的时间内获得一定的收 益, 但是单独某一分位的收益实际波动很大, 扣除手续费用之后的收益更少了。那么我们该 如何修正已有的模型来构造更为稳定且收益较好的模型呢?

\section{4. 对模型进行修正以构造投资组合}

本文所研究的，其一是为了检验基于中证 800 研究中国股市存在动量与反转效应的存在， 当然这个在上面我们已经得到了验证; 其二就是构造更为稳定的投资组合。有着较高的收益, 不能持续，不是大多投资者所愿意投资的，只有持续的超额收益才是投资者所愿投资的项目。

\section{1 模型修正}

从前文的研究中我们可以看出我们在5-5策略上按传统的等量划分股票为5分位模型中的 第一分位比Li, Pan等人所研究的模型好, 但是我最后的收益好坏不是投资模型的唯一选择标 
准, 我们应该知道传统模型中各期的收益率是否都能比 $\mathrm{Li}, \mathrm{Pan}$ 等人构造的模型更好, 是否收 益更为稳定。下图为5-5策略第一分位中两种模型各期收益率曲线:

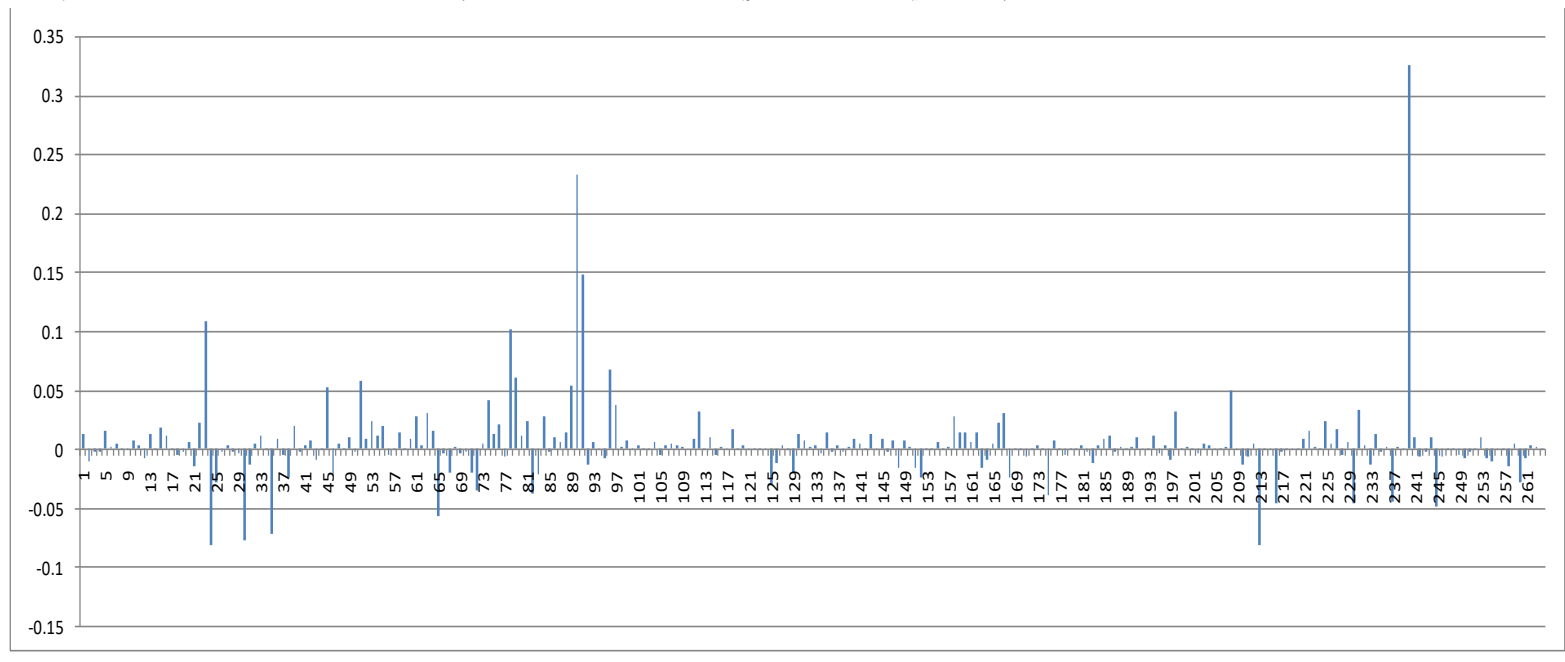

图1 5-5策略第一分位中模型各期收益率曲线

由上图我们的结果表示: 收益差为正数占比为 $57 \%$, 表明传统模型并不存在总是超越 $\mathrm{Li}$, $P a n$ 等人所研究的按收益率等量划分为 5 分位的模型, 但是它有着更为稳定的收益, 可以算出 两种模型各期收益率的方差分别为0.056624和0.066933, 因而按等量划分股票持有第一分位的 收益率更好。但是我们还是不能简单这样判断持有传统模型的第一分位就是最好的, 因为短 期操作越频繁，交易的手续费越高，在按等量划分股票5-5策略中我们共交易了264次，按每 次交易 $0.5 \%$ 的费用计算，其费用高达 3.731129 , 这对于我们的收益影响很大，我们期望根据 对分位的权重重新分配或者加以多空策略后获得更好的收益;

\section{2 投资组合构建}

$\mathrm{Li}, \mathrm{Pan}$ 等人所构造的模型中20-20策略存在优于其他样本期检验期组合的收益，也存在期 间收益的最大值, 但是其最后收益最大值存在于第一分位, 却是第五分位存在期间最大值, 所以，对于各自两个分位存在较大的波动，我们将基于多空组合将对其进行检验。

3.3.1 基于两种模型, 分别对于5-5策略的第一分位股票赋予权重

对进入每个分位中的股票在样本期的收益率进行排序（从大到小或从小到大），利用衰 减因子构造的权重系数:

$$
\omega_{t}=\frac{(1-\lambda) \lambda^{\wedge}(t-1)}{1-\lambda^{\wedge} T}(t=1,2,3, \ldots, T)
$$

（注： $\lambda$ 一般取 $0.9, \mathrm{~T}$ 为每个分位的股票总数）

将构造出的权重与相对应的各个股票相配对，得出各个分位的最终收益：

$$
f(x)=\prod_{n=1}^{N} \sum_{t=1}^{T}\left(1+\omega_{t} R_{t n}\right)
$$

加权重后的收益并没有想象中的那么好，对于等量划分股票或者等量划分收益率效果都 不好, 下图为样本期和检验期都为 5 天的两种策略下加以权重后的收益图。不仅收益的极值变 小了，同时其投资趋势依旧波动很大，没有达到减小风险的效果。 


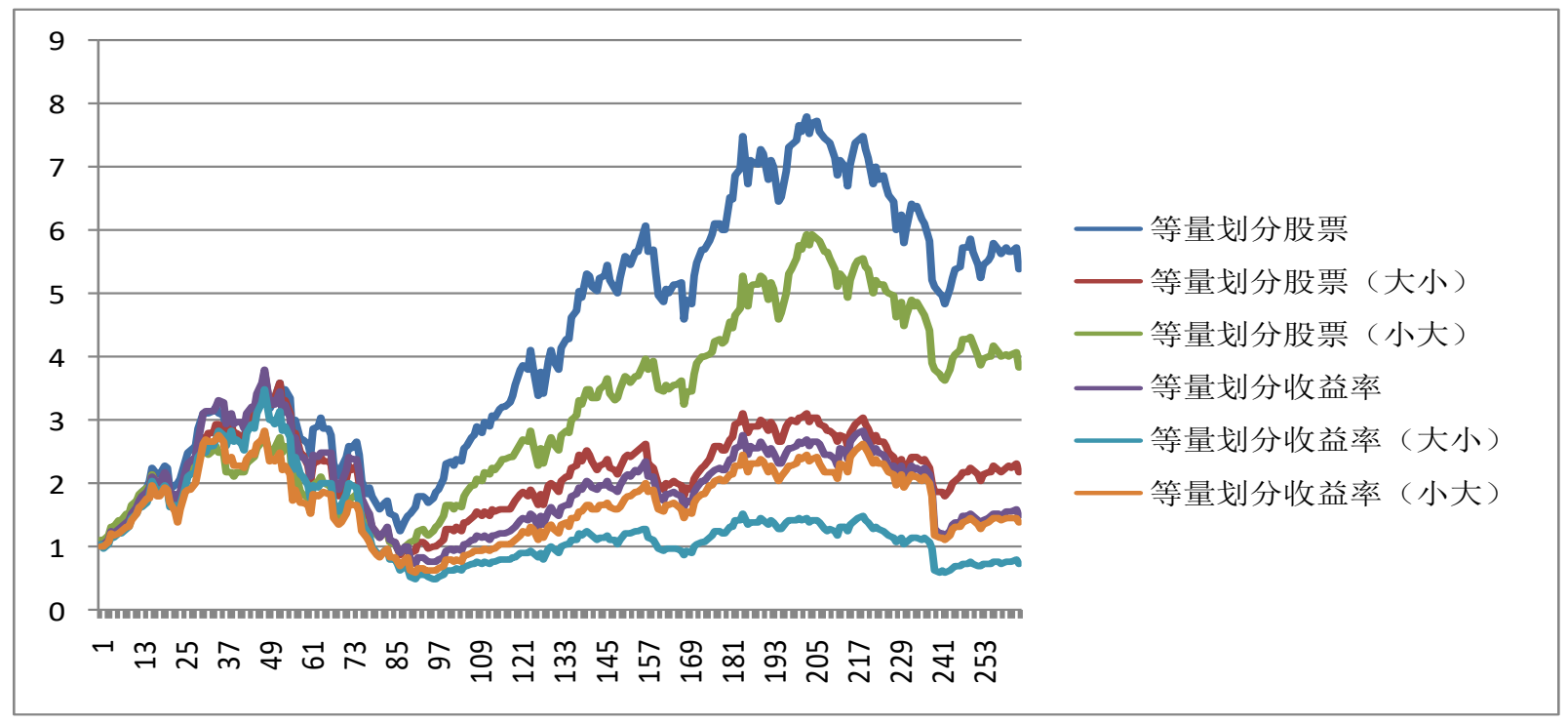

图2 赋予权重后投资策略的收益率

\subsection{2 构建多空策略投资组合}

Jegadeesh和 Titman (1993)提出的J-K模型，其多空策略在理论上很有借鉴意义。从上面 的分析中我们知道, 而且是在 $\mathrm{Li}, \mathrm{Pan}$ 等人的模型上, 单独一个分位很难获得良好的收益, 既 然股市存在反转效应和动量效应, 那么我们是否能在短期操作中基于反转运用多空策略以降 低风险获得更好的收益呢，而在长期中基于动量运用多头获得收益。虽然我国多空策略具有 一定的局限性，但是在2010年首次融资融券试点的启动，以及近期2013年2月28日的转融通业 务终于实现, 都表明做空时代即将到来, 所以多空策略尤其存在的必然性意义。我们按多空 比重150/50检验两种策略中5-5,20-20的效果，同时对于20-20策略只采用多头策略，但只有等 量划分股票5-5策略150/50多空策略能持续获得较好的收益, 其他组合都没有这个效果。此时, 我们再将交易费用考虑进来，即在每期交易中扣除 $0.5 \%$ 的交易费用，我们在初始投资 1 块钱最 终能涨到 5.4282 块钱, 折算为年收益 $36.0109 \%$ 。所以, 在中国股市存在较强的反转效应的情 况下，基于中证800按等量划分股票5-5策略能获得较为满意且稳定的收益。



图3 等量划分股票5-5策略150/50多空策略收益率

\section{4. 结论:}

本文以中证800为研究对象, 选取2007年1月15号至2012年6月29号的日收盘价数据, 对中 国股市的进行动量与反转检验, 结果发现中国股市存在较强的短期反转效应, 而存在较弱的 长期动量效应。 
此外本文使用 $\mathrm{Li}$ ，Pan等人基于按等收益划分为五分位的模型检验中证500是否依旧存在 较好的投资机会, 结果发现Li, Pan等人的模型并不适应在中证800上的运用, 因为这个模型并 没有达到像选择对象为全部A股那样具有持续的收益。为了抓住这个动量反转效应, 把握相 应的投资机会, 我们选择构造传统的按等量划分股票模型, 同时构造多空组合, 最后检验出 传统的按等量划分股票的5-5策略加以150/50的多空权重, 能获得较为出色的收益。

\section{References}

[1] Wang $\mathrm{Pu}$, Momentum effect and contrarian in China stock market, Shaanxi Journal of Agricultural Sciences, vol.57, pp. 184-186, 2011.

[2] Yan Taihua, Liang Lan, The research on momentum effect of Shanghai stock market_—base on weekly return data from1995 to 2009, Technology Economics, vol.30, pp. 109-114, 2011.

[3] Bondt W F M, Thaler R, Does the stock market overreact, The Journal of finance, vol.40, pp. 793-805, 1985.

[4] Lehmann B, Fads, martingales, and market efficiency, Quarterly Journal of Economics, vol.105, pp. 1-28, 1988.

[5] Pan L, Tang Y, Xu J, Weekly momentum by return interval ranking, Pacific-Basin Finance Journal, vol.21, pp. 1191-1208, 2013.

[6] Jegadeesh N, Titman S, Returns to buying winners and selling losers: Implications for stock market efficiency, The Journal of finance, vol.48, pp. 65-91, 1993.

[7] Sandy, Lee. Review of 2011 Asia Quantitative Outlook and introduction of new ideas, London: London, 2011. 